\title{
5 Wie wählt man den geeigneten Operateur aus?
}

Grundsätzlich gilt: Führt eine Klinik eine bestimme Behandlung und ein Operateur eine bestimmte Operation häufig aus, sichert dies mit höherer Wahrscheinlichkeit ein gutes Ergebnis. Die persönliche Routine und Erfahrung eines Operateurs ist sehr wichtig.

Sie brauchen daher einen Arzt, der sich langjährig mit dem Kniegelenk beschäftigt hat, daneben aber den gesamten Bewegungsapparat überblickt und auch Krankheitsbilder aus benachbarten Fachgebieten abgrenzen kann. Die Politik hat aus diesem Grunde Mindestfallzahlen in einigen Bereichen eingeführt. Auch geben spezielle Zertifizierungen (z.B. Endoprothesenzentrum der Maximalversorgung) Auskunft über bestandene Qualitätsaudits der Ärzte und Kliniken. Trauen Sie sich durchaus, den potentiellen Operateur direkt zu fragen, wie viel Erfahrung er speziell in Ihrem Fall hat!

Aber nicht nur der Arzt als Operateur ist entscheidend; das gesamte Team einschließlich der Anästhesieabteilung, das gut aufeinander abgestimmt ist und über eine große Routine verfügt, garantiert ein gutes Ergebnis. Schließlich sollte auch die Infrastruktur des Krankenhauses alles Notwendige bieten, wie z.B. modern ausgestattete postoperative Überwachungseinheiten (InterMediate Care, IMC).

Bei der Auswahl der Klinik können Patientenforen und Klinikbewertungsportale im Internet informativ sein; die Bewertungen sind jedoch subjektiv und keinesfalls repräsentativ. Die wohl verlässlichsten Informationen bieten die von den Kliniken veröffentlichten Qualitätsberichte.

\section{WebTipp}

Hilfe bei der Kliniksuche erhalten Sie z. B. unter www.tk.de/tk/klinikfuehrer/114928 oder www.weisse-liste.de. 